\title{
Correction to: Investigations of Ag nanosol impact on the nonlinear optical properties of neutral red dye
}

\author{
Bini P. Pathrose ${ }^{1}$ Anitha Prakash ${ }^{1}$ V. P. N. Nampoori ${ }^{1}$ • \\ P. Radhakrishnan ${ }^{1}$ A. Mujeeb ${ }^{1}$
}

\section{Correction to: Opt Quant Electron (2018)50:26 https://doi.org/10.1007/s11082-017-1300-4}

The article title was incorrectly published in the original publication. The correct title is given in this erratum.

The original article can be found online at https://doi.org/10.1007/s11082-017-1300-4.

Bini P. Pathrose

binivibin@gmail.com

Anitha Prakash

anuprakash.55@gmail.com

V. P. N. Nampoori

vpnnampoori@cusat.ac.in

P. Radhakrishnan

radhak@cusat.ac.in

A. Mujeeb

mujeebpoovar@gmail.com

1 International School of Photonics, Cochin University of Science and Technology, Cochin, Kerala, India 